\title{
Differences in Treatment and Outcome of Pancreatic Adenocarcinoma Stage I and II in the EURECCA Pancreas Consortium
}

\author{
J. V. Groen ${ }^{1}$, B. G. Sibinga Mulder', E. van Eycken ${ }^{2}$, Z. Valerianova ${ }^{3}$, J. M. Borras ${ }^{4}$, L. G. M. van der Geest ${ }^{5}$, \\ G. Capretti ${ }^{6}$, A. Schlesinger-Raab ${ }^{7}$, M. Primic-Zakelj, ${ }^{8}$ A. Ryzhov ${ }^{9}$, C. J. H. van de Velde ${ }^{1}$, B. A. Bonsing ${ }^{1}$, \\ E. Bastiaannet ${ }^{1}$, and J. S. D. Mieog ${ }^{1}$
}

${ }^{1}$ Department of Surgery, Leiden University Medical Center, Leiden, The Netherlands; ${ }^{2}$ Belgian Cancer Registry, Brussels, Belgium; ${ }^{3}$ Bulgarian National Cancer Registry/National Oncological Hospital, Sofia, Bulgaria; ${ }^{4}$ Department of Clinical Sciences, University of Barcelona, Barcelona, Spain; ${ }^{5}$ Department of Research, Netherlands Comprehensive Cancer Organisation (IKNL), Utrecht, The Netherlands; ${ }^{6}$ Pancreatic Surgery Unit, Department of Surgery, Humanitas University, Milan, Italy; ${ }^{7}$ Munich Cancer Registry, Institute for Medical Information Processing, Biometry and Epidemiology, Ludwig-Maximilians-University, Munich, Germany; ${ }^{8}$ Epidemiology and Cancer Registry/Institute of Oncology Ljubljana, Ljubljana, Slovenia; ${ }^{9}$ Taras Shevchenko National University of Kyiv and Ukrainian National Cancer Institute, Kiev, Ukraine

\begin{abstract}
Background. The EUropean REgistration of Cancer CAre (EURECCA) consortium aims to investigate differences in treatment and to improve cancer care through Europe. The purpose of this study was to compare neo- and adjuvant chemotherapy (ACT) and outcome after tumor resection for pancreatic adenocarcinoma stage I and II in the EURECCA Pancreas consortium.

Methods. The eight, collaborating national, regional, and single-center partners shared their anonymized dataset. Patients diagnosed in 2012-2013 who underwent tumor resection for pancreatic adenocarcinoma stage I and II were investigated with respect to treatment and survival and compared using uni- and multivariable logistic and Cox regression analyses. All comparisons were performed separately per registry type: national, regional, and singlecenter registries.
\end{abstract}

Electronic supplementary material The online version of this article (https://doi.org/10.1245/s10434-018-6705-1) contains supplementary material, which is available to authorized users.

(C) The Author(s) 2018

First Received: 14 March 2018; Published Online: 27 August 2018

J. S. D. Mieog

e-mail: J.S.D.Mieog@lumc.nl
Results. In total, 2052 patients were included. Stage II was present in the majority of patients. The use of neo-ACT was limited in most registries (range $2.8-15.5 \%$ ) and was only different between Belgium and The Netherlands after adjustment for potential confounders. The use of ACT was different between the registries (range 40.5-70.0\%), even after adjustment for potential confounders. Ninety-day mortality was also different between the registries (range $0.9-13.6 \%$ ). In multivariable analyses for overall survival, differences were observed between the national and regional registries. Furthermore, patients in ascending age groups and patients with stage II showed a significant worse overall survival.

Conclusions. This study provides a clear insight in clinical practice in the EURECCA Pancreas consortium. The differences observed in (neo-)ACT and outcome give us the chance to further investigate the best practices and improve outcome of pancreatic adenocarcinoma.

Pancreatic cancer (PC) is one of the few types of cancer with increasing incidence and mortality rates. ${ }^{1}$ In 2017 , the number of annual deaths in the European Union due to PC will exceed the number of death due to breast cancer. ${ }^{2}$ Resection is the only chance for prolonged survival; unfortunately only $15-20 \%$ of PC patients are eligible for resection due to advanced or metastatic disease at diagnosis. ${ }^{3}$ Tumor/node/metastases (TNM) stage I and II PC 
are generally considered eligible for resection. ${ }^{4}$ The European Society of Medical Oncology (ESMO) guidelines, during the study period and most recent, state that patients with a borderline resectable or locally advanced tumor should be treated with neoadjuvant chemotherapy (neo-ACT) in clinical trials whenever possible and that adjuvant chemotherapy (ACT) is considered as standard of care after curative resection for PC. ${ }^{5,6}$ Recently, the ESPAC-4 trial showed a survival benefit in patients treated with adjuvant gemcitabine and capecitabine compared with gemcitabine alone. ${ }^{7}$ Despite advances in (neo)-ACT, the median survival for patients with an initial resectable tumor is only 23.3 (range 12-54) months. ${ }^{8}$

Previous studies have reported variations in incidence, mortality and survival in PC between countries. ${ }^{9-12}$ The EUropean REgistration of Cancer CAre (EURECCA) consortium, established by the European CanCer Organisation (ECCO), aims to investigate differences in treatment and to improve cancer care through Europe. ${ }^{13}$ International comparisons of (neo-)ACT and outcome in surgically treated patients with PC are sparse. Therefore, the purpose of this study was to describe and compare (neo-)ACT and outcome of patients who underwent tumor resection for resectable (TNM stage I and II) pancreatic adenocarcinoma in the EURECCA Pancreas consortium.

\section{MATERIALS AND METHODS}

\section{Study Design and Data Preparation}

This is an observational cohort study of eight partners (registries) in the EURECCA Pancreas consortium [national: Belgium (BE), The Netherlands (NL), Slovenia (SLO), Ukraine (UA), and Bulgaria (BG); regional: Catalonia (Spain) (CAT(E)) and Munich (Germany) (MU(D); and single center: Milan (Italy) (MIL(I))] who shared their anonymized dataset. Detailed description of the registries is provided in Table S1 (Supplementary). The American Joint Committee on Cancer and International Union Against Cancer TNM 7th Edition classification were used to describe stage. ${ }^{4,14}$ In case pathology TNM variables were not informative (missing or X), clinical TNM variables were used as replacement. In case clinical TNM variables also were not informative (missing or $\mathrm{X}$ ), pathology TNM variables were considered to be " 0 ." The 3rd edition of the International Classification of Disease for Oncology was used for topographical and morphological (i.e., pathologic diagnosis) coding. ${ }^{15}$ Age was categorized as $<65$ years, $65-75$ years, and $>75$ years. Overall survival (OS) was calculated from date of surgery until date of death (event) or last follow-up (censored). Ninety-day mortality was calculated to distinguish surgery-related from disease-related death. ${ }^{16}$

\section{Patient Selection}

All patients with pancreatic tumors (included codes: C25.0-C25.9; excluded: C25.4), diagnosed in 2012-2013 (present in all registries), undergoing tumor resection, for adenocarcinoma (included codes: 8140-8380, 8500-8585; excluded: $8150-8158,8240-8249)$, stage I and II were included. ${ }^{15}$ Patients with a history of other malignancies were not excluded, because PC is most often determinative for the prognosis. BG could not confirm tumor resection and was only used in descriptive statistics in Table S2 (Supplementary). SLO and UA were not included in analyses of neo-ACT, because no information was available. CAT(E) and UA were not included in analyses of ACT, because no information was available.

\section{Statistical Analyses}

Statistical analyses were performed using SPSS Inc. for Windows (version 23.0). Numerical data are reported as mean [standard deviation (SD)] and compared using the one-way ANOVA test. Categorical data are reported as absolute numbers (percentages) and compared using the Chi square test. Multivariable logistics regression analyses (adjusted for sex, age group, and stage) where performed for neo-ACT, ACT, and 90-day mortality. Kaplan-Meier curves, log-rank tests, and multivariable Cox regression analyses (adjusted for sex, age group, stage) where used to compare OS. For multivariable comparisons between registries, BE (national) and CAT(E) (regional) were used as reference groups (first in alphabetic order). For reasons of bias, comparisons were performed separately per registry type: national, regional, and single-center registries. To assess the risk of missing data bias, sensitivity analyses were conducted by adding patients with "unknown" stage to the original analyses. To assess the influence of 90-day mortality on the use of ACT, multivariable sensitivity analysis was performed with 90-day mortality as covariate. To assess the influence of use of (neo-)ACT on OS, multivariable sensitivity analysis was performed with (neo-)ACT as covariates. The original results were considered robust if the sensitivity analyses showed similar results. $P<0.05$ was considered statistically significant for all analyses.

\section{RESULTS}

\section{Patient and Tumor Characteristics}

Figure S1 (Supplementary) illustrates the inclusion of patients in this study. In total, 2052 patients diagnosed in 2012-2013 underwent tumor resection for pancreatic adenocarcinoma stage I and II were included (Table 1). 
TABLE 1 Patient and tumor characteristics

\begin{tabular}{|c|c|c|c|c|c|c|c|c|c|c|c|c|c|c|c|c|}
\hline & \multicolumn{16}{|c|}{ Registry } \\
\hline & \multicolumn{8}{|c|}{ National } & \multirow[t]{3}{*}{$P$ value } & \multicolumn{4}{|c|}{ Regional } & \multirow[t]{3}{*}{$P$ value } & \multirow{2}{*}{\multicolumn{2}{|c|}{$\begin{array}{l}\begin{array}{l}\text { Single } \\
\text { center }\end{array} \\
\begin{array}{l}\text { Milan } \\
(N=110)\end{array}\end{array}$}} \\
\hline & \multicolumn{2}{|c|}{$\begin{array}{l}\text { Belgium } \\
(N=469)\end{array}$} & \multicolumn{2}{|c|}{$\begin{array}{l}\text { The } \\
\text { Netherlands } \\
(N=645)\end{array}$} & \multicolumn{2}{|c|}{$\begin{array}{l}\text { Slovenia } \\
(N=73)\end{array}$} & \multicolumn{2}{|c|}{$\begin{array}{l}\text { Ukraine } \\
(N=214)\end{array}$} & & \multicolumn{2}{|c|}{$\begin{array}{l}\text { Catalonia } \\
(N=210)\end{array}$} & \multicolumn{2}{|c|}{$\begin{array}{l}\text { Munich } \\
(N=331)\end{array}$} & & & \\
\hline & $N$ & $\%$ & $N$ & $\%$ & $N$ & $\%$ & $N$ & $\%$ & & $N$ & $\%$ & $N$ & $\%$ & & $N$ & $\%$ \\
\hline \multicolumn{17}{|l|}{ Sex } \\
\hline Male & 256 & $54.6 \%$ & 329 & $51.0 \%$ & 39 & $53.4 \%$ & 130 & $60.7 \%$ & 0.098 & 116 & $55.2 \%$ & 161 & $48.6 \%$ & 0.135 & 60 & $54.5 \%$ \\
\hline Female & 213 & $45.4 \%$ & 316 & $49.0 \%$ & 34 & $46.6 \%$ & 84 & $39.3 \%$ & & 94 & $44.8 \%$ & 170 & $51.4 \%$ & & 50 & $45.5 \%$ \\
\hline \multicolumn{17}{|l|}{ Age } \\
\hline Mean (SD) & \multicolumn{2}{|c|}{$66.7(10.0)$} & \multicolumn{2}{|c|}{$66.0(9.0)$} & \multicolumn{2}{|c|}{$65.6(10.2)$} & \multicolumn{2}{|c|}{$57.5(9.8)$} & $<0.001$ & \multicolumn{2}{|c|}{$67.4(9.6)$} & \multicolumn{2}{|c|}{$69.3(9.2)$} & 0.020 & \multicolumn{2}{|c|}{$68.3(9.8)$} \\
\hline \multicolumn{17}{|l|}{ Stage } \\
\hline I & 70 & $14.9 \%$ & 65 & $10.1 \%$ & 6 & $8.2 \%$ & 46 & $21.5 \%$ & $<0.001$ & 20 & $9.5 \%$ & 10 & $3.0 \%$ & 0.001 & 2 & $1.8 \%$ \\
\hline II & 399 & $85.1 \%$ & 580 & $89.9 \%$ & 67 & $91.8 \%$ & 168 & $78.5 \%$ & & 190 & $90.5 \%$ & 321 & $97.0 \%$ & & 108 & $98.2 \%$ \\
\hline \multicolumn{17}{|l|}{ Location } \\
\hline Head of pancreas & 287 & $61.2 \%$ & 525 & $81.4 \%$ & 56 & $76.7 \%$ & 145 & $67.8 \%$ & $<0.001$ & 176 & $83.8 \%$ & 252 & $76.1 \%$ & $<0.001$ & 70 & $63.6 \%$ \\
\hline Body of pancreas & 25 & $5.3 \%$ & 18 & $2.8 \%$ & 8 & $11.0 \%$ & 20 & $9.3 \%$ & & 27 & $12.9 \%$ & 16 & $4.8 \%$ & & 0 & $0.0 \%$ \\
\hline Tail of pancreas & 35 & $7.5 \%$ & 47 & $7.3 \%$ & 6 & $8.2 \%$ & 16 & $7.5 \%$ & & 7 & $3.3 \%$ & 27 & $8.2 \%$ & & 0 & $0.0 \%$ \\
\hline Other pancreas & 122 & $26.0 \%$ & 55 & $8.5 \%$ & 3 & $4.1 \%$ & 33 & $15.4 \%$ & & 0 & $0.0 \%$ & 36 & $10.9 \%$ & & $40^{1}$ & $36.4 \%$ \\
\hline \multicolumn{17}{|l|}{ Type of surgery } \\
\hline Pancreatoduodenectomy & 377 & $80.4 \%$ & 571 & $88.5 \%$ & 0 & $0.0 \%$ & 149 & $69.6 \%$ & $<0.001$ & 200 & $95.2 \%$ & 240 & $72.5 \%$ & $<0.001$ & 70 & $63.6 \%$ \\
\hline Other ${ }^{2}$ & 92 & $19.6 \%$ & 73 & $11.3 \%$ & 0 & $0.0 \%$ & 65 & $30.4 \%$ & & 10 & $4.8 \%$ & 91 & $27.5 \%$ & & 40 & $36.4 \%$ \\
\hline Unknown & 0 & $0.0 \%$ & 1 & $0.2 \%$ & $73^{3}$ & $100.0 \%$ & 0 & $0.0 \%$ & & 0 & $0.0 \%$ & 0 & $0.0 \%$ & & 0 & $0.0 \%$ \\
\hline
\end{tabular}

${ }^{1}$ Includes tumours from body and tail of pancreas

${ }^{2}$ Other types of pancreatectomy (e.g., total and distal pancreatectomy or enucleation)

${ }^{3}$ Authors confirmed these patients underwent oncological resections

Distribution of males/females was largely comparable between the registries. The mean (SD) age differed between the national registries, ranging from 57.5 (11.8) years in UA to 66.7 (10.0) years in BE, and the regional registries, 67.4 (9.6) years in CAT(E) and 69.3 (9.2) years in MU(D). In all registries, stage II patients were the majority of patients undergoing tumor resection, ranging from $78.5 \%$ (UA) to $98.2 \%$ (MIL(I)). Overall, tumors were most often $(73.6 \%)$ located in "head of pancreas" and "pancreatoduodenectomy" was performed in majority (81.2\%) of patients, excluding SLO who did not specify type of resection. Table S2 (Supplementary) shows characteristics of patients for BG, who could not confirm tumor resection.

\section{Neoadjuvant Chemotherapy}

Overall, the use of neo-ACT ranged from $2.8 \%$ in NL to $15.5 \%$ in MIL(I). There were no differences between the national and regional registries (Figs. 1a, b).

Multivariable analyses showed differences in odds ratios (OR) for the use of neo-ACT between the national registries: patients in NL were less likely to receive neo-ACT compared with $\mathrm{BE}$ (NL: odds ratio $[\mathrm{OR}]=0.48,95 \%$ confidence interval $[\mathrm{CI}]=0.29-0.89, P=0.020$; Table 2). No other predictive factors where identified in the national, regional, or single-center registries. Sensitivity analyses with patients with unknown stage added to the multivariable analyses showed similar OR.

\section{Adjuvant Chemotherapy}

Overall, the use of ACT ranged from $40.5 \%$ in MU(D) to $70.0 \%$ in MIL(I). A higher proportion of ACT in stage II versus stage I was observed in all registries (Figs. 1c, d). The proportion of patients with stage II receiving ACT varied between the national registries $(P=0.017)$.

Multivariable analyses showed considerable differences in OR for the use of ACT between the national registries (Table 2). Patients in NL and SLO were significantly less likely to receive ACT compared with $\mathrm{BE}(\mathrm{NL}: \mathrm{OR}=0.70$, 95\% CI $=0.53-0.93, P=0.012 ;$ SLO: $\mathrm{OR}=0.32,95 \%$ $\mathrm{CI}=0.19-0.56, \quad P<0.001) . \quad$ Furthermore, patients in ascending age group and patients with stage I were less likely to receive ACT in the national registries. In the 


\section{a Neoadjuvant chemotherapy in stage I}

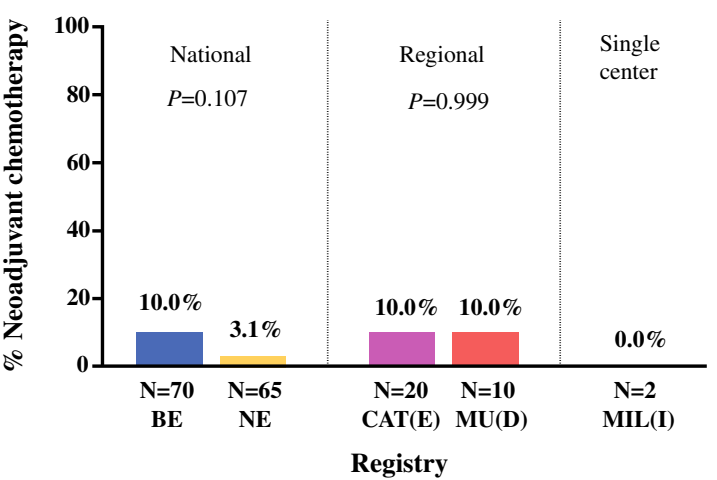

c

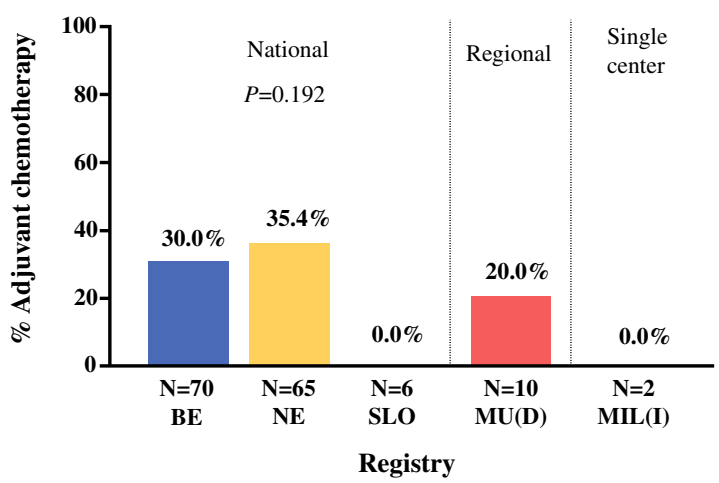

\section{b Neoadjuvant chemotherapy in stage II}

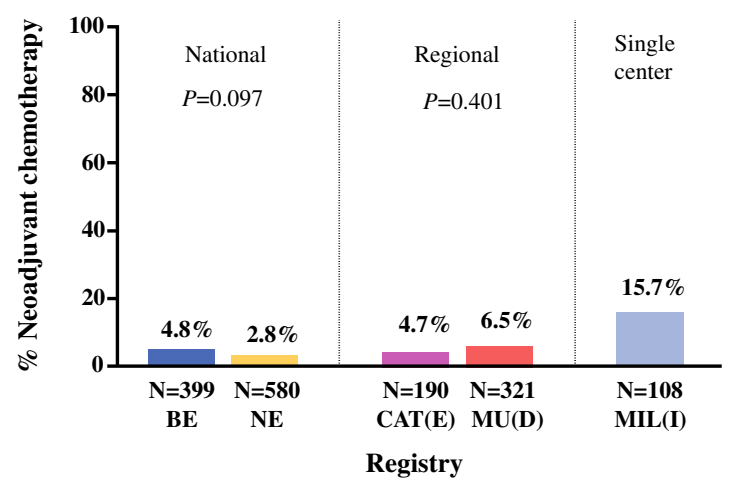

d Adjuvant chemotherapy in stage II

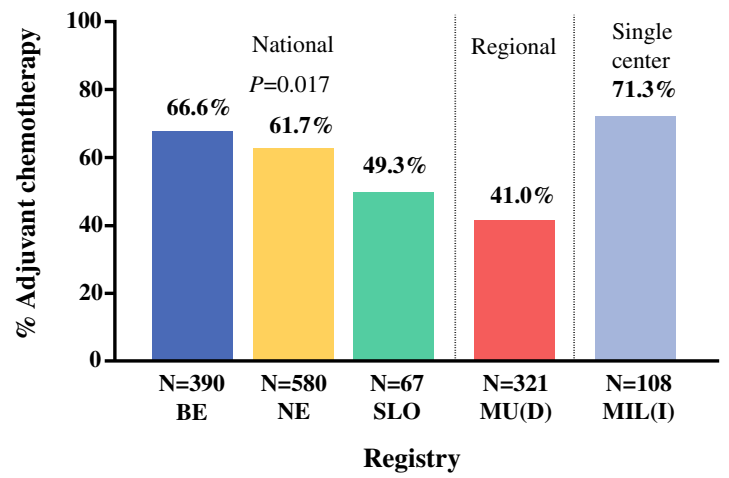

FIG. 1 Neoadjuvant and adjuvant chemotherapy per registry in a neoadjuvant chemotherapy in stage I, b neoadjuvant chemotherapy in stage II, c adjuvant chemotherapy in stage I, $\mathbf{d}$ adjuvant chemotherapy in stage II

regional and single-center registry, patients in age group $>75$ years also were less likely to receive ACT. Sensitivity analyses with patients with unknown stage added to the multivariable analyses showed similar results, except that in regional and single-center registries each ascending age group was significantly less likely to receive ACT. Sensitivity analyses with 90-day mortality as covariate in the multivariable analyses showed similar OR.

\section{Ninety-Day Mortality}

Ninety-day mortality differed between the national registries $(P=0.001 ; \quad$ Fig. 2). UA (13.6\%) and MU(D) (8.5\%) had the highest 90-day mortality in the national and regional registries respectively, whereas overall MIL(I) (single-center registry) had the lowest 90-day mortality $(0.9 \%)$.

Multivariable analyses showed considerable differences in OR for 90-day mortality between the national registries (Table 2). Compared with BE, patients in NL had lower 90-day mortality $(\mathrm{OR}=0.56, \quad 95 \% \quad \mathrm{CI}=0.35-0.89$, $P=0.014)$ and patients in $\mathrm{UA}(\mathrm{OR}=2.21,95 \% \mathrm{CI}=$ 1.23-3.68, $P=0.007$ ) had higher 90-day mortality.
Female and the younger age group were significant protective factors for 90-day mortality in the national registries. No predictive factors were identified in the regional registries. Multivariable analyses in the singlecenter registry was not possible due to a low number of events. Sensitivity analyses with patients with unknown stage added to the multivariable analyses showed similar OR.

\section{Overall Survival}

OS was significantly different in the national $(P<0.001)$ and regional $(P=0.005)$ registries (Fig. 3a, c). In multivariable analysis for OS in the national registries, UA showed a significantly different OS compared with $\mathrm{BE}$ (hazard ratio $(\mathrm{HR})=2.29,95 \% \mathrm{CI}=1.83-2.85$, $P<0.001$; Table 2). Female sex was a significant protective factors for $\mathrm{OS}(\mathrm{HR}=0.77,95 \% \mathrm{CI}=0.68-0.87$, $P<0.001)$. Patients in each ascending age group (65-75 years: $\quad \mathrm{HR}=1.16, \quad 95 \% \quad \mathrm{CI}=1.01-1.34$, $P=0.040 ;>75$ years: $H R=1.75,95 \% \mathrm{CI}=1.44-2.12$, $P<0.001)$ and stage II $(\mathrm{HR}=1.86,95 \% \mathrm{CI}=1.69-2.31$, $P<0.001)$ showed worse OS. In the regional registries, 


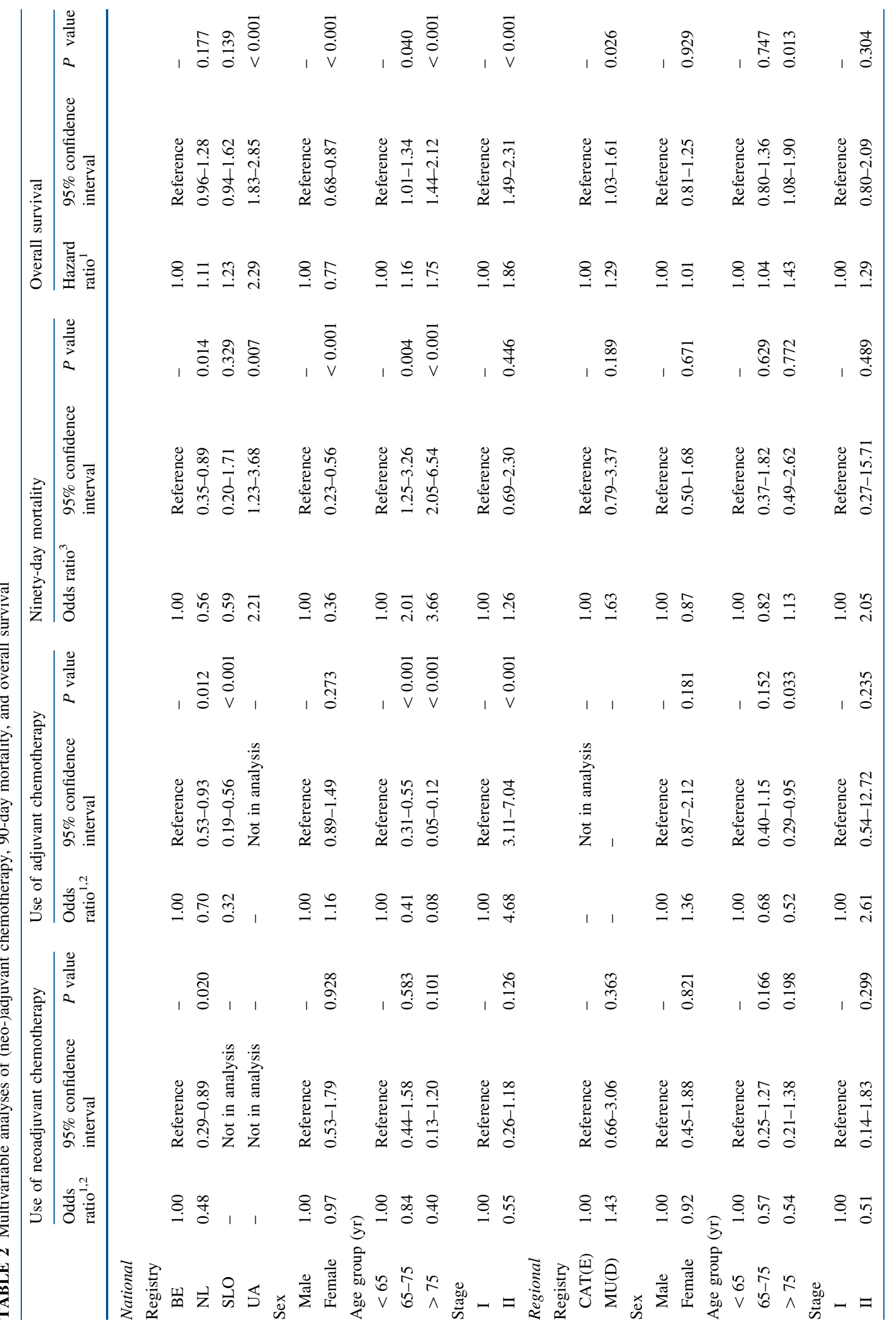




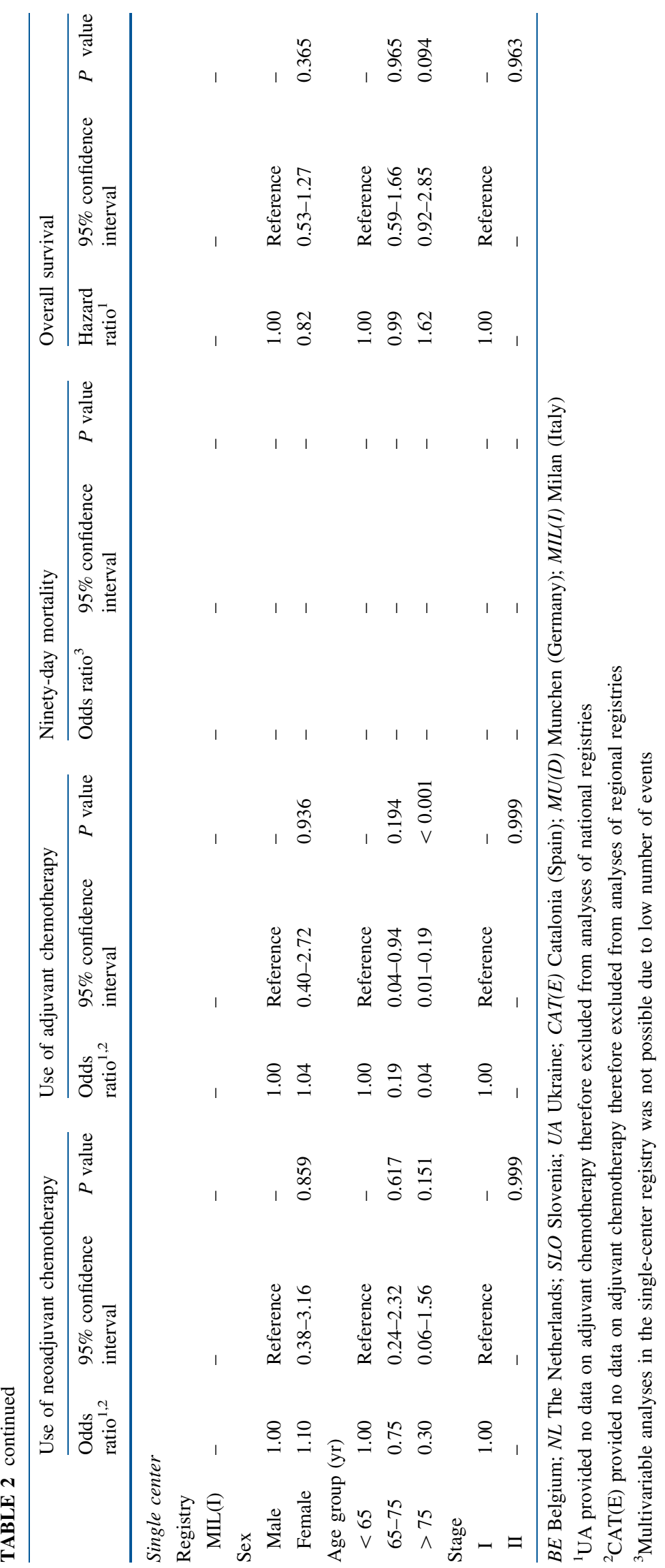


MU(D) showed a significantly different OS compared with CAT(E) $\quad(\mathrm{HR}=1.29,95 \% \mathrm{CI}=1.03-1.61, P=0.026)$. Age group $>75$ years was a significant factor with worse OS compared to age group $<65$ years $(\mathrm{HR}=1.43,95 \%$ $\mathrm{CI}=1.08-1.90, \quad P=0.013), \quad$ whereas the age group 65-75 years was not. Also, sex and stage were not significant factors for OS. In the single-center registry, only the age group $>75$ years was a borderline significant factor with worse OS compared with the age group $<65$ years $(\mathrm{HR}=1.62,95 \% \mathrm{CI}=0.92-2.85, P=0.094)$.

In addition, median $(95 \% \mathrm{CI})$ survival of patients who received ACT was: 20.1 (18.5-21.7) months in the national-, 19.0 (15.6-22.4) months in the regional-, and 30.0 (24.4-35.6) months in the single center registries and median $(95 \% \mathrm{CI})$ survival of ACT naïve patients: 12.1 (10.3-13.9) months in the national-, 14.0 (11.2-16.8) months in the regional-, and 19.0 (11.1-26.8) months in the single center registries, although a direct comparison is not possible.

Sensitivity analyses with patients with unknown stage added to the multivariable analyses showed similar HR.

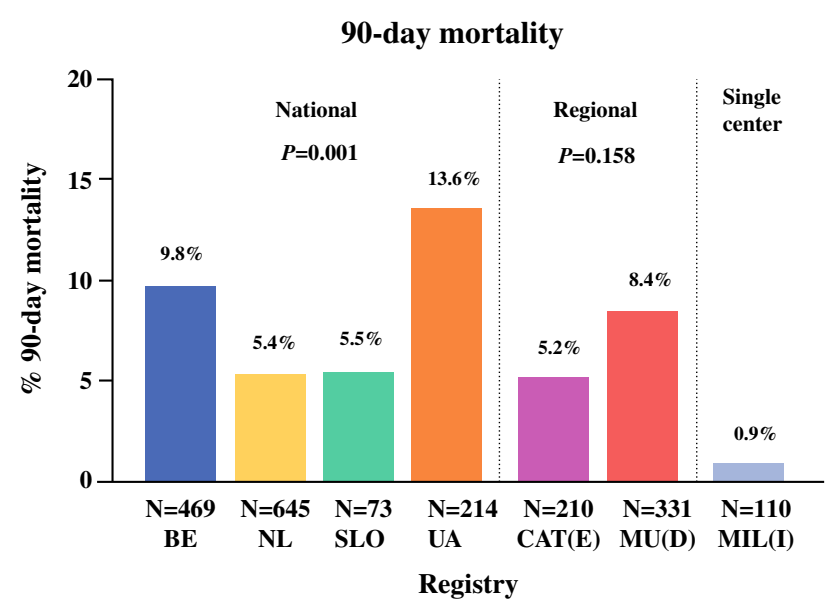

FIG. 2 Ninety-day mortality rates per registry
Sensitivity analyses with ACT added to the multivariable analyses showed similar HR.

\section{DISCUSSION}

The main purpose of this study was to describe and compare (neo-)ACT and outcomes of patients who underwent tumor resection for stage I and II pancreatic adenocarcinoma in the EURECCA consortium. There were some differences in the use of neo-ACT. Although the ESMO guidelines-during the study period and most recently-recommended the use of ACT, variations were observed in OR for ACT usage between national registries. ${ }^{6}$ Also, large variations in 90-day mortality and OS were observed between the registries included in this study.

Previous studies from the EURECCA consortium showed variations in the use of chemo(radiation)therapy in colon, rectal, and breast cancer patients. ${ }^{17-19}$ The observed variations in neo-ACT, but mainly ACT, between the registries in this study are in concordance with a recent large-scale international study of resected PC patients. ${ }^{20} \mathrm{~A}$ possible explanation for the variations can be differences in adherence to (inter)national guidelines. ${ }^{18,19}$ Also, cultural, socioeconomic, and healthcare differences may play a role in the use of (neo-)ACT. ${ }^{21-23}$ The observation that few patients received neo-ACT was probably due to the statement by the ESMO guidelines (during the study period) that neo-ACT should be used in clinical trial settings. ${ }^{6}$ Clinical trials are more easily accessible in specialized centers, which explains the greater use of neo-ACT in the (specialized) single-center registry compared with the national and regional registries. A recent meta-analysis has shown the benefit of neo-ACT over upfront surgery. ${ }^{24}$ An interesting international comparison would be how these results are implemented in more recent practice. A complicated postoperative course can delay or omit the use of $\mathrm{ACT}^{25}$ In a sensitivity analyses with 90-day mortality added to the multivariable analyses for the use of ACT, we confirmed that differences in 90-day mortality were not of

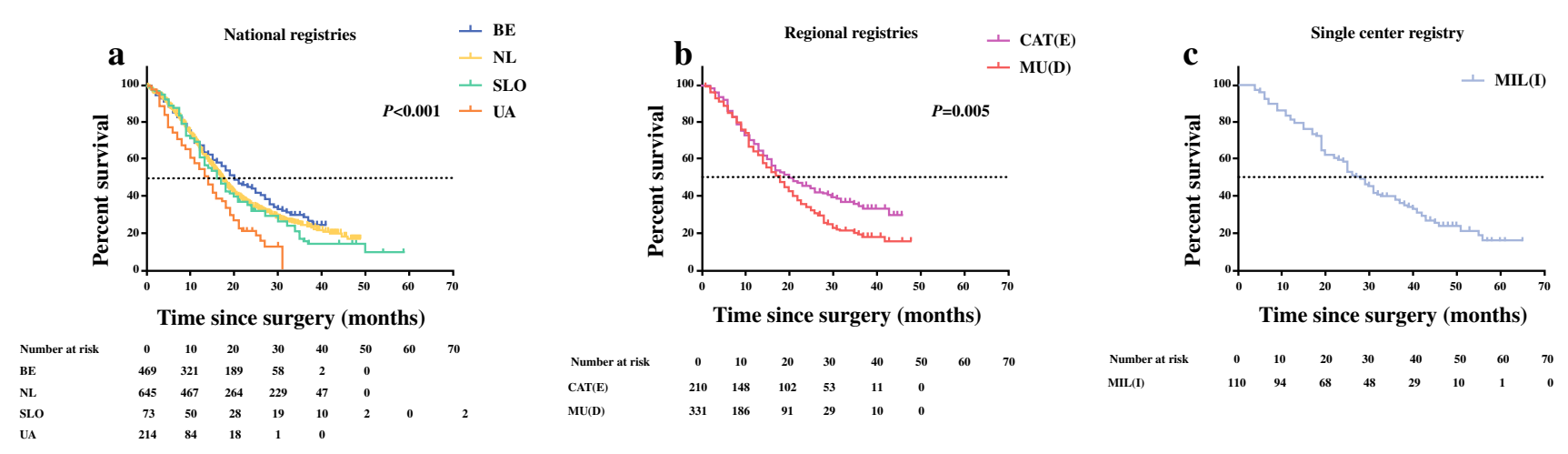

FIG. 3 Kaplan-Meier curves of overall survival per registry: a national registries, b regional registries, and $\mathbf{c}$ single-center registry 
influence on the differences in the use of ACT between the registries. The use of ACT decreased per ascending age group and patients in the age group $>75$ years showed a significant worse OS in multivariable analyses in the national, regional, and single-center registries. As previously investigated, elderly patients are at higher risk of postoperative complications. ${ }^{26}$ Although centralization improved outcome of pancreatic surgery in elderly patients in a recent study, further research is needed to gain knowledge on this matter. ${ }^{27}$

Variations in 90-day mortality were observed between the national registries, even after adjustment for sex, age group, and stage. Multiple studies have shown a lower postoperative mortality after pancreatic surgery in highcompared with low-volume hospitals. ${ }^{28,29}$ In our study this could not be assessed, because the annual hospital volumes were not available. Nonetheless, BE and MU(D) showed a high 90-day mortality and centralization of pancreatic surgery was not (yet) implemented over there during the study period. Caution has to be taken with this statement as detailed information about perioperative treatment, likely to affect 90-day mortality, was not available.

This study showed a better survival in patients receiving ACT compared with naïve patients in the national, regional, and single-center registries. This can be explained by confounding by indication (fit patients with a good prognosis are generally more likely to receive ACT), and therefore a justifiable comparison is not possible. The recent ESPAC-4 trial (2017) showed a significant better survival for patients treated with adjuvant gemcitabine and capecitabine compared with gemcitabine alone $(28.0$ (95\% $\mathrm{CI}=23.5-31.5$ months $\quad$ vs. $25.5(95 \% \quad \mathrm{CI}=22.7-27.9)$ months) after resection for PC. ${ }^{7}$ Considering the randomized ESPAC-trial has strict inclusion criteria (e.g., full recovery after surgery, creatinine clearance $\geq 50 \mathrm{~mL} / \mathrm{min}$ ) and our study is mainly population-based, the results are largely comparable. Still, direct comparison is hampered by the differences in study design. In a sensitivity analyses with (neo-)ACT added to the multivariable analyses for OS, we confirmed that differences in ACT were not of influence on the differences in OS between the registries. Definite conclusions cannot be drawn from this sensitivity analysis, because immortal time bias and confounding by indication cannot be ruled out.

Our study has several limitations. First, caution has to be taken with interpretation of the results as differences in (unmeasured) patient characteristics (e.g., patient selection for tumor resection) might have been of influence. Nevertheless, analyses were adjusted for important factors (sex, age group, stage) and still showed differences between the registries. Second, due to inherent differences between national, regional, and single-center registries, which also explain the observed inter-registry-type variations, analyses had to be performed separately per registry type and lowered the statistical power (e.g., multivariable analyses for 90-day mortality was not possible in the single-center registry). Third, due to missing data this study excluded some patients (e.g., unknown stage or tumor resection) and registries (e.g., SLO and UA did not provide data on neo-ACT, CAT(E), and UA did not provide data on $\mathrm{ACT}$ and the dataset from BG could not confirm tumor resection) from certain analyses. A possible explanation for this is that the provided datasets may originally have been established for other intentions (e.g., Cancer Registry or Clinical/Surgical Audit) and thus focused on completeness of certain (other) variables. Although most included registries are surgically driven and therefore very comparable, this probably introduced missing data bias. ${ }^{30}$ Sensitivity analyses with patients with unknown stage added to the analyses confirmed the robustness of the results of this study. Still, variables, such as stage and tumor resection, are pivotal when investigating treatment and outcome in cancer patients. Future registration should focus on completeness and uniform use of definitions as previously stated by other member of the EURECCA consortium. ${ }^{13,17}$ Nonetheless, this study is the first to describe and compare (neo-)ACT and outcome of patients undergoing tumor resection for pancreatic adenocarcinoma stage I and II in eight different European registries.

\section{CONCLUSIONS}

The results of this study give a clear insight in the clinical practice of the partners in the EURECCA Pancreas consortium. Overall, the variations illustrate the difference in implementation of universally accepted and used guidelines for treatment of pancreatic adenocarcinoma stage I and II. The differences in the use of (neo-)ACT and outcome provide us the chance to further investigate the best practices. Moreover, the EURECCA Pancreas consortium underlines the need for uniform registration as international comparisons will become increasingly important pillars of international guidelines.

ACKNOWLEDGMENT The authors thank Dr. De Schutter and Mrs. Verbeeck (Belgian Cancer Registry, Brussels, Belgium), Dr. Manchon-Wals (Cancer Plan, Barcelona), and the Munich Cancer Registry for the collection of data. The authors also thank the registration team of the Netherlands Comprehensive Cancer Organisation (IKNL) for the collection of data for the Netherlands Cancer Registry as well as IKNL staff for scientific advice. Furthermore, the authors thank Prof. Zerbi (Pancreatic Surgery Unit, Department of Surgery, Humanitas University, Milan, Italy) for revising the manuscript.

FUNDING EURECCA was funded by the European Society of Surgical Oncology. This work was supported by the Bas Mulder Award from the Alpe d'HuZes foundation/Dutch Cancer Society 
[UL2015-7665 to J.V.G., B.G.S.M. and J.S.D.M.]. The funding sources had no role in the study design, collection, analyses, interpretation of the data, drafting of the manuscript, or the decision to publish.

PREVIOUS COMMUNICATION "Minimal Standards and Quality Assurance in Oncology: Focus on treatment of solid tumors," Padova, Italy, October 2017.

SOURCES OF SUPPORT EURECCA was funded by the European Society of Surgical Oncology. This work was supported by the Bas Mulder Award (Grant UL2015-7665) from the Alpe d'HuZes foundation/Dutch Cancer Society.

CONFLICT OF INTEREST All authors declare that they have no conflict of interest.

OPEN ACCESS This article is distributed under the terms of the Creative Commons Attribution 4.0 International License (http://crea tivecommons.org/licenses/by/4.0/), which permits unrestricted use, distribution, and reproduction in any medium, provided you give appropriate credit to the original author(s) and the source, provide a link to the Creative Commons license, and indicate if changes were made.

\section{REFERENCES}

1. Siegel RL, Miller KD, Jemal A. Cancer statistics, 2016. $C A$ Cancer J Clin. 2016;66(1):7-30.

2. Ferlay J, Partensky C, Bray F. More deaths from pancreatic cancer than breast cancer in the EU by 2017. Acta Oncol. 2016;55(9-10):1158-60.

3. Ryan DP, Hong TS, Bardeesy N. Pancreatic adenocarcinoma. N Engl J Med. 2014;371(22):2140-1.

4. Edge SB, Compton CC. The American Joint Committee on Cancer: the 7th edition of the AJCC cancer staging manual and the future of TNM. Ann Surg Oncol. 2010;17(6):1471-4.

5. Ducreux M, Cuhna AS, Caramella C, et al. Cancer of the pancreas: ESMO Clinical Practice Guidelines for diagnosis, treatment and follow-up. Ann Oncol. 2015;26 Suppl 5:v56-68.

6. Seufferlein T, Bachet JB, Van Cutsem E, Rougier P, Group EGW. Pancreatic adenocarcinoma: ESMO-ESDO Clinical Practice Guidelines for diagnosis, treatment and follow-up. Ann Oncol. 2012;23 Suppl 7:vii33-40.

7. Neoptolemos JP, Palmer DH, Ghaneh P, et al. Comparison of adjuvant gemcitabine and capecitabine with gemcitabine monotherapy in patients with resected pancreatic cancer (ESPAC-4): a multicentre, open-label, randomised, phase 3 trial. Lancet. 2017;389(10073):1011-24.

8. Gillen S, Schuster T, Meyer Zum Buschenfelde C, Friess H, Kleeff J. Preoperative/neoadjuvant therapy in pancreatic cancer: a systematic review and meta-analysis of response and resection percentages. PLoS Med. 2010;7(4):e1000267.

9. Lepage C, Capocaccia R, Hackl M, et al. Survival in patients with primary liver cancer, gallbladder and extrahepatic biliary tract cancer and pancreatic cancer in Europe 1999-2007: results of EUROCARE-5. Eur J Cancer. 2015;51(15):2169-78.

10. Karim-Kos HE, de Vries E, Soerjomataram I, Lemmens V, Siesling S, Coebergh JW. Recent trends of cancer in Europe: a combined approach of incidence, survival and mortality for 17 cancer sites since the 1990s. Eur J Cancer. 2008;44(10):1345-89.
11. Ferlay J, Soerjomataram I, Dikshit R, et al. Cancer incidence and mortality worldwide: sources, methods and major patterns in GLOBOCAN 2012. Int J Cancer. 2015;136(5):E359-86.

12. Bosetti C, Bertuccio P, Malvezzi M, et al. Cancer mortality in Europe, 2005-2009, and an overview of trends since 1980. Ann Oncol. 2013;24(10):2657-71.

13. van Gijn W, van de Velde CJ, members of the Ec. Improving quality of cancer care through surgical audit. Eur J Surg Oncol. 2010;36 Suppl 1:S23-6.

14. L.H. Sobin MKG, Ch. Wittekind. TNM classification of malignant tumours, 7th edn. Geneva, Switzerland: International Union Against Cancer. 2009.

15. World-Health-Organization. International Classification of Diseases for Oncology (ICD-O-3) (3rd edn). 2013:http://codes.iarc.f r/. Accessed 18 Sept 2017.

16. Mise Y, Vauthey JN, Zimmitti G, et al. Ninety-day postoperative mortality is a legitimate measure of hepatopancreatobiliary surgical quality. Ann Surg. 2015;262(6):1071-8.

17. Breugom AJ, Bastiaannet E, Boelens PG, et al. Adjuvant chemotherapy and relative survival of patients with stage II colon cancer - A EURECCA international comparison between the Netherlands, Denmark, Sweden, England, Ireland, Belgium, and Lithuania. Eur J Cancer. 2016;63:110-7.

18. Kiderlen M, Walsh PM, Bastiaannet E, et al. Treatment strategies and survival of older breast cancer patients - an international comparison between the Netherlands and Ireland. PLoS One. 2015;10(2):e0118074.

19. van den Broek CB, van Gijn W, Bastiaannet E, et al. Differences in pre-operative treatment for rectal cancer between Norway, Sweden, Denmark, Belgium and the Netherlands. Eur J Surg Oncol. 2014;40(12):1789-96.

20. Huang L, Jansen L, Balavarca Y, et al. Resection of pancreatic cancer in Europe and USA: an international large-scale study highlighting large variations. Gut. Nov 202017.

21. Cheung MC, Yang R, Byrne MM, Solorzano CC, Nakeeb A, Koniaris LG. Are patients of low socioeconomic status receiving suboptimal management for pancreatic adenocarcinoma? Cancer. 2010;116(3):723-33.

22. Shapiro M, Chen Q, Huang Q, et al. Associations of socioeconomic variables with resection, stage, and survival in patients with early-stage pancreatic cancer. JAMA Surg. 2016;151(4):338-45.

23. van Roest MH, van der Aa MA, van der Geest LG, de Jong KP. The impact of socioeconomic status, surgical resection and type of hospital on survival in patients with pancreatic cancer. a population-based study in The Netherlands. PLoS One. 2016;11(11):e0166449.

24. Versteijne E, Vogel JA, Besselink MG, et al. Meta-analysis comparing upfront surgery with neoadjuvant treatment in patients with resectable or borderline resectable pancreatic cancer. $\mathrm{Br} \mathrm{J}$ Surg. Apr 302018.

25. McMillan MT, Vollmer CM, Jr., Asbun HJ, et al. The characterization and prediction of ISGPF grade $\mathrm{C}$ fistulas following pancreatoduodenectomy. J Gastrointest Surg. 2016;20(2):262-76.

26. Sukharamwala P, Thoens J, Szuchmacher M, Smith J, DeVito P. Advanced age is a risk factor for post-operative complications and mortality after a pancreaticoduodenectomy: a meta-analysis and systematic review. HPB (Oxford). 2012;14(10):649-57.

27. van der Geest LG, Besselink MG, Busch OR, et al. Elderly patients strongly benefit from centralization of pancreatic cancer surgery: a population-based study. Ann Surg Oncol. 2016;23(6):2002-9.

28. Krautz C, Nimptsch U, Weber GF, Mansky T, Grutzmann R. Effect of hospital volume on in-hospital morbidity and mortality following pancreatic surgery in Germany. Ann Surg. Apr 04 2017. 
29. Balzano G, Zerbi A, Capretti G, Rocchetti S, Capitanio V, Di Carlo V. Effect of hospital volume on outcome of pancreaticoduodenectomy in Italy. Br J Surg. 2008;95(3):357-62
30. Westreich D. Berkson's bias, selection bias, and missing data. Epidemiology. 2012;23(1):159-64. 